\title{
Mortality Outcomes of Patients with Chronic Kidney Disease and Chronic Obstructive Pulmonary Disease
}

\author{
Sankar D. Navaneethan ${ }^{a, b}$ Jesse D. Schold ${ }^{c, d}$ Haiquan Huang ${ }^{c, g}$ \\ Georges Nakhoul $^{c}$ Stacey E. Jolly ${ }^{e}$ Susana Arrigain ${ }^{d}$ Raed A. Dweik $^{f}$ \\ Joseph V. Nally Jrc \\ a Selzman Institute for Kidney Health, Section of Nephrology, Department of Medicine, Baylor College of Medicine,

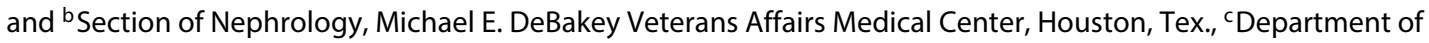 \\ Nephrology and Hypertension, Glickman Urological and Kidney Institute, d Department of Quantitative Health \\ Sciences, ${ }^{\mathrm{e}}$ Medicine Institute, and ${ }^{\mathrm{f}}$ Respiratory Institute, Cleveland Clinic, Cleveland, Ohio, USA; ${ }^{9}$ Department of \\ Geriatric, Zhongda Hospital Affiliated with Southeast University, Nanjing, China
}

\section{Key Words}

Chronic obstructive pulmonary disease - Death and kidney disease $\cdot$ Cause-specific death $\cdot$ Lung disease

\footnotetext{
Abstract

Background: Chronic obstructive pulmonary disease (COPD) is associated with higher mortality in the general population. We studied the associations between COPD and death among chronic kidney disease (CKD) patients along with reporting cause-specific death data. Methods: We included 56,960 patients with stages 3 and 4 CKD who were followed in a large health care system. Associations between COPD and all-cause mortality and various causes of death (respiratory deaths, cardiovascular deaths, malignancy-related deaths and deaths due to other reasons) were studied using the Cox proportional hazards and competing risk models. Results: Out of 56,960 CKD patients, $4.7 \%(n=2,667)$ had underlying COPD. Old age, presence of diabetes, hypertension, coronary artery disease, congestive heart failure, and smoking were associated with higher risk for COPD. During a median follow-up of 3.7 years, 15,969 patients died. After covariate adjustment, COPD was associated with a $41 \%$ in-
}

creased risk (95\% Cl 1.31-1.52) for all-cause mortality, and fourfold increased risk (sub-hazard ratio $4.36,95 \% \mathrm{Cl} 3.54-$ 5.37) for respiratory-related deaths. In a sensitivity analysis that was performed by defining COPD as the use of relevant International Classification of Diseases-9 codes and medications used to treat COPD, similar results were noted. Conclusions: COPD is associated with higher risk for death among those with CKD, and an underlying lung disease accounts for significant proportion of deaths. These data highlight the need for further prospective studies to understand the underlying mechanisms and potential interventions to improve outcomes in this population.

() 2016 S. Karger AG, Basel

\section{Background}

Chronic obstructive pulmonary disease (COPD) is an important public health problem and is predicted to become the third leading cause of death [1]. Chronic kidney disease $(\mathrm{CKD})$ is also an emerging public health issue around the globe. The prevalence of both CKD and COPD increases with age and both these diseases are associated with atherosclerotic disease $[2,3]$. While the mechanisms

\section{KARGER}

E-Mail karger@karger.com

www.karger.com/ajn
(C) 2016 S. Karger AG, Basel

0250-8095/16/0431-0039\$39.50/0
Sankar D. Navaneethan, MD, MS, MPH

Selzman Institute for Kidney Health, Section of Nephrology

Baylor College of Medicine, 1 Baylor Plaza, Suite 100.37D

Houston, TX 77030 (USA)

E-Mail Sankar.navaneethan@bcm.edu 
linking CKD and COPD have not been completely elucidated, systemic inflammation and hypoxia associated with COPD could contribute to adverse outcomes in those with CKD [4].

Despite the clinical significance of these 2 diseases, a limited number of studies have examined their associations. The reported prevalence of kidney disease in COPD ranges from 20 to $30 \%[5,6]$. Chandra et al. [7] reported that estimated glomerular filtration rate (eGFR) and serum creatinine correlated with the severity of emphysema noted on CT scan, but not with the severity of airflow obstruction by spirometry. In a cohort of 3,371 patients undergoing vascular surgery, CKD was noted in $27 \%$ and COPD in $39 \%$ and the prevalence of COPD increased with the reduction in eGFR. In long-term follow-up, moderate and severe COPD were associated with increased mortality among those with CKD [5]. However, another study found no significant association between airflow obstruction and CKD after adjusting for confounding variables [8]. Importantly, studies examining the impact of COPD in those with CKD, particularly on death and causes of death are lacking. Hence, we studied the factors associated with the presence of COPD and the associations of COPD with all-cause mortality among a non-dialysis CKD population followed in a large health care system. In addition, we also report the various causes of deaths in this population.

\section{Methods}

This analysis was conducted using a pre-existing electronic health record (EHR)-based CKD registry at the Cleveland Clinic. The development and validation of the EHR-based CKD registry at the Cleveland Clinic has been described in detail elsewhere [9].

\section{Study Population}

Patients who met the following criteria from January 1, 2005 through December 31, 2012, and lived in the state of Ohio were included: (1) had at least one face-to-face outpatient encounter with a Cleveland Clinic health care provider and (2) had at least 2 or more eGFR $15-59.9 \mathrm{ml} / \mathrm{min} / 1.73 \mathrm{~m}^{2}$ that were at least 90 days apart (using the CKD epidemiology collaboration (CKDEPI) equation) [9]. Patients with lung transplant or resection, end-stage renal disease needing dialysis, and renal transplant prior to CKD diagnosis were excluded. Patients outside the state of Ohio were excluded as cause-specific death data was not available.

\section{Definitions and Outcome Measures}

Variables

Demographic details were extracted from the EHR. Diabetes mellitus, hypertension, coronary artery disease, and other comorbidities were defined using pre-specified criteria and validated.
Relevant outpatient laboratory values were obtained from the EHR. Medication details were obtained from the EHR and were validated by one of the investigators.

Renal Function

We applied the CKD-EPI equation to patients who had 2 outpatient serum creatinine levels between January 1, 2005 and December 31, 2012, to calculate eGFR. All creatinine measurements were performed by the modified kinetic Jaffe reaction, using a Hitachi D 2400 Modular Chemistry Analyzer (Roche Diagnostics, Indianapolis, Ind., USA) in our laboratory. CKD was defined according to current guidelines as follows: stage 3 CKD (eGFR $30-59 \mathrm{ml} / \mathrm{min} / 1.73 \mathrm{~m}^{2}$ ) and stage 4 CKD (eGFR 15-29 ml/ $\min / 1.73 \mathrm{~m}^{2}$ ). We further categorized stage 3 into CKD stage $3 \mathrm{a}$ (eGFR $45-59 \mathrm{ml} / \mathrm{min} / 1.73 \mathrm{~m}^{2}$ ) and stage $3 \mathrm{~b}$ (eGFR $30-44 \mathrm{ml} /$ $\left.\min / 1.73 \mathrm{~m}^{2}\right)$.

\section{COPD}

COPD was defined as the presence of 2 International Classification of Diseases (ICD), Ninth Revision (ICD-9) codes in any encounter type in the EHR. A modified definition of COPD and bronchiectasis as recommended by the Agency for Healthcare Research and Quality was used and included the following ICD-9 codes: 491, 491.0, 491.1, 491.2, 491.20, 491.21, 491.22, 491.8, 491.9, $492,492.0,492.8,494,494.0,494.1,496$. In a sensitivity analysis, we also defined COPD as the use of abovementioned ICD-9 codes plus the use of relevant medications used to manage COPD.

\section{Mortality}

The primary outcome of interest, all-cause mortality, was ascertained from our EHR and linkage of the CKD registry with the Ohio Department of Health mortality data. We censored patients on December 2012. For cause-specific death analysis, we used the Ohio Department of Health mortality data. The underlying cause of death was coded according to the ICD, Tenth Revision (ICD$10)$. We grouped the underlying causes of death as per the National Center for Health Statistics for each coding system, except for some changes as outlined below. We classified deaths into the following 4 major categories: (a) cardiovascular deaths, (b) malignancy, (c) respiratory, and (d) other deaths. We defined cardiovascular deaths as deaths due to diseases of the heart, essential hypertension, cerebrovascular disease, atherosclerosis, or other diseases of the circulatory system (ICD-10 codes I00-I78). Respiratory deaths were defined using the ICD-10 codes J40-J47.

\section{Statistical Analysis}

We compared baseline characteristics between patients with and without COPD using chi-square and t tests for categorical and continuous variables, respectively. We used a logistic regression model to evaluate the factors associated with having COPD. We included the following variables: age, race, gender, BMI group, eGFR, diabetes, malignancy, coronary artery disease, congestive heart failure, hypertension, and smoking while adjusting for year of entry into the registry. We used a Kaplan-Meier curve and the log-rank test to evaluate the relationship between COPD and mortality. We also used unadjusted and adjusted Cox proportional hazards models to evaluate the relationship between COPD and all-cause mortality and competing risks regression analysis to evaluate the relationship between COPD and various causes of death. The mortality models were adjusted for age, gender, race, CKD stage, diabetes, hyperten-
40

Am J Nephrol 2016;43:39-46

DOI: $10.1159 / 000444422$
Navaneethan/Schold/Huang/Nakhoul/ Jolly/Arrigain/Dweik/Nally Jr 
sion, hyperlipidemia, malignancy, insurance, smoking, coronary artery disease, congestive heart failure, cerebrovascular disease, peripheral vascular disease, BMI group, angiotensin converting enzyme/angiotensin receptor blocker (ACE/ARB) use, beta blocker use, and statin use, albumin, hemoglobin, and current prescription of COPD medications. Inception point for survival analysis was the date of the second eGFR $<60 \mathrm{ml} / \mathrm{min} / 1.73 \mathrm{~m}^{2}$.

We tested two-way interactions between CKD and age, race, gender and CKD stage on overall mortality. Because there were some patients with COPD and no prior prescription of COPD medications, we also did a sensitivity analysis defining COPD as the presence of relevant ICD-9 codes plus prior prescription of COPD medications while excluding patients with COPD diagnosis and no prior prescription.

The percentage of missing information among patients was as follows: $3 \%$ insurance information, $15 \%$ smoking status, $5 \% \mathrm{BMI}$, $19 \%$ albumin, and $18 \%$ hemoglobin. We used multiple imputations (SAS proc MI) with the Markov Chain Monte Carlo method and a single chain to impute 5 datasets with complete continuous and binary covariate data in a first step, and then in a second step, we imputed insurance group on each of the 5 datasets using discriminant function analysis. All logistic and Cox models were performed on each of the 5 imputed datasets, and parameter estimates were combined using SAS MIanalyze. To evaluate the effect of using multiple imputations, we fit the logistic, Cox, and competing risks models on complete cases only. We also tabulated the leading causes of death in those with and without COPD (online suppl. table S1; for all online suppl. material, see www.karger.com/ doi/10.1159/000444422).

All analyses were conducted using Unix SAS version 9.4 (SAS Institute, Cary, N.C., USA), and graphs were created using R 3.0.1 (The R Foundation for Statistical Computing, Vienna, Austria). The CKD registry and this study were approved by the Cleveland Clinic Institutional Review Board.

\section{Results}

\section{Baseline Patient Characteristics}

Between January 1, 2005 and December 31, 2012, 63,560 patients were included in our CKD registry with eGFR $15-$ $59 \mathrm{ml} / \mathrm{min} / 1.73 \mathrm{~m}^{2}$. We excluded 6,600 patients because they did not meet the specified inclusion criteria (online suppl. fig. S1). The clinical characteristics of the patients with and without COPD are presented in table 1. Patients with COPD were older and had a higher proportion of comorbid conditions such as diabetes, hyperlipidemia, coronary artery disease, and congestive heart failure (table 1).

\section{Factors Associated with COPD}

In the multivariable logistic regression model, older age, African American race, diabetes, coronary artery disease, congestive heart failure, hypertension and smoking were associated with statistically and significantly higher odds of having COPD (table 2).

\section{COPD and All-Cause Mortality}

With a median follow-up of 3.7 years, there were 15,969 deaths. The Kaplan-Meier analysis (fig. 1) showed significant differences in all-cause mortality for CKD patients with and without COPD. After adjusting for age, gender, race, CKD stage, diabetes, hypertension, hyperlipidemia, malignancy, insurance, smoking, coronary artery disease, congestive heart failure, cerebrovascular disease, peripheral vascular disease, BMI group, ACE/ARB use, beta blocker use, and statin use, albumin, hemoglobin, and current prescription of COPD medications, COPD was associated with significantly higher hazards of mortality (hazard ratio 1.41 95\% CI 1.31-1.52, $\mathrm{p}<0.05$; fig. 2).

\section{Causes of Death}

Causes of death details were available from the Ohio Department of Health mortality data for 15,761 patients. We excluded the small number $(n=208)$ of patients whose deaths were only found in our EHR from all causespecific mortality analyses. Online supplemental table 1 shows the causes of death overall and by COPD. Figure 2 shows the associations between various causes of death in those with COPD and highlights that the underlying respiratory diseases poses a higher sub-hazard than other causes.

\section{Sensitivity Analysis}

Complete Case Analysis

Results similar to primary analyses were noted in a sensitivity analysis restricting those with complete details (table 3).

COPD Defined as Use of ICD-9 Codes and

Bronchodilators

The sensitivity analysis defining COPD as those with ICD-9 diagnoses codes along with the use of COPD medication, while excluding from analysis patients with ICD9 diagnosis codes and no medications, yielded similar results (table 3 ).

\section{Interactions}

The interaction between COPD and age on overall mortality was significant $(\mathrm{p}=0.001)$, indicating that the hazard associated with COPD is stronger among younger patients. The interaction between COPD and gender on overall mortality was also significant, indicating that the hazard associated with COPD is significant for both genders but stronger among females (table 4). The interactions between COPD and race, and COPD and CKD stage were not significant. 
Table 1. Characteristics of study cohort based on the presence or absence of COPD

\begin{tabular}{|c|c|c|c|c|c|}
\hline Factor & $\mathrm{n}$ & Overall & $\begin{array}{l}\text { No COPD } \\
(\mathrm{n}=54,293)\end{array}$ & $\begin{array}{l}\text { COPD } \\
(n=2,667)\end{array}$ & $\mathrm{p}$ value \\
\hline Age & 56,960 & $72.4 \pm 11.8$ & $72.2 \pm 11.8$ & $74.6 \pm 9.7$ & $<0.001^{\mathrm{a}}$ \\
\hline Male, gender, $\%$ & 56,960 & 43.7 & 43.6 & 46.0 & $0.017^{\mathrm{b}}$ \\
\hline African American, \% & 56,960 & 12.5 & 12.4 & 15.1 & $<0.001^{\mathrm{b}}$ \\
\hline Smoking, \% & 56,960 & & & & $<0.001^{\mathrm{b}}$ \\
\hline No & & 77.9 & 77.8 & 79.8 & \\
\hline Yes & & 7.0 & 6.5 & 16.8 & \\
\hline Missing & & 15.1 & 15.7 & 3.4 & \\
\hline $\mathrm{BMI}, \mathrm{kg} / \mathrm{m}^{2}$ & 54,144 & $29.5 \pm 6.6$ & $29.5 \pm 6.5$ & $29.1 \pm 7.1$ & $<0.001^{\mathrm{a}}$ \\
\hline BMI group, $\mathrm{kg} / \mathrm{m}^{2}$ & 56,960 & & & & $<0.001^{\mathrm{b}}$ \\
\hline$<18.5$ & & 1.2 & 1.1 & 2.8 & \\
\hline $18.5-24.9$ & & 22.6 & 22.4 & 26.3 & \\
\hline $25-29.9$ & & 33.8 & 34.0 & 30.8 & \\
\hline $30+$ & & 37.4 & 37.5 & 36.7 & \\
\hline Missing & & 4.9 & 5.0 & 3.4 & \\
\hline Diabetes, \% & 56,960 & 22.8 & 22.5 & 29.7 & $<0.001^{\mathrm{b}}$ \\
\hline Malignancy, \% & 56,960 & 24.4 & 24.2 & 26.8 & $0.003^{\mathrm{b}}$ \\
\hline Hypertension, \% & 56,960 & 84.5 & 84.0 & 95.2 & $<0.001^{\mathrm{b}}$ \\
\hline Hyperlipidemia, \% & 56,960 & 76.9 & 76.5 & 84.3 & $<0.001^{\mathrm{b}}$ \\
\hline Coronary artery disease, $\%$ & 56,960 & 20.2 & 19.5 & 34.4 & $<0.001^{\mathrm{b}}$ \\
\hline Congestive heart failure, $\%$ & 56,960 & 7.8 & 7.2 & 18.4 & $<0.001^{\mathrm{b}}$ \\
\hline Cerebrovascular disease, $\%$ & 56,960 & 9.0 & 8.7 & 14.7 & $<0.001^{\mathrm{b}}$ \\
\hline $\mathrm{ACE} / \mathrm{ARB}$ use, $\%$ & 56,960 & 63.2 & 62.7 & 72.7 & $<0.001^{\mathrm{b}}$ \\
\hline Statins use, \% & 56,960 & 57.0 & 56.6 & 66.6 & $<0.001^{\mathrm{b}}$ \\
\hline$\beta$-Blocker use, $\%$ & 56,960 & 57.4 & 57.2 & 62.4 & $<0.001^{\mathrm{b}}$ \\
\hline $\mathrm{eGFR}, \mathrm{ml} / \mathrm{min} / 1.73 \mathrm{~m}^{2}$ & 56,960 & $47.6 \pm 10.3$ & $47.6 \pm 10.3$ & $47.7 \pm 9.9$ & $0.75^{\mathrm{a}}$ \\
\hline eGFR categories, $\mathrm{ml} / \mathrm{min} / 1.73 \mathrm{~m}^{2}$ & 56,960 & & & & $0.52^{\mathrm{b}}$ \\
\hline $45-59$ & & 67.4 & 67.3 & 67.7 & \\
\hline $30-44$ & & 24.9 & 24.9 & 25.1 & \\
\hline $15-29$ & & 7.8 & 7.8 & 7.2 & \\
\hline Albumin, g/dl & 46,137 & $4.1 \pm 0.79$ & $4.1 \pm 0.80$ & $4.1 \pm 0.42$ & $0.86^{\mathrm{a}}$ \\
\hline Hemoglobin, mg/dl & 46,963 & $12.8 \pm 1.8$ & $12.8 \pm 1.8$ & $12.7 \pm 1.9$ & $0.012^{\mathrm{a}}$ \\
\hline Insurance, $\%$ & 56,960 & & & & $<0.001^{\mathrm{b}}$ \\
\hline Medicaid & & 0.72 & 0.70 & 1.2 & \\
\hline Medicare & & 78.7 & 78.4 & 84.6 & \\
\hline Missing & & 3.0 & 3.1 & 2.2 & \\
\hline Other & & 17.5 & 17.8 & 12.0 & \\
\hline Bronchodilator use, \% & 56,960 & 30.0 & 27.1 & 88.5 & $<0.001^{\mathrm{b}}$ \\
\hline
\end{tabular}

\section{Discussion}

In this study of stages 3 and $4 \mathrm{CKD}$ patients who are followed in a large health care system, COPD (defined using ICD-9 codes) was prevalent in $5.3 \%$ of CKD population. Old age and multiple comorbid conditions were associated with higher odds of COPD. Even though modest, the presence of COPD was associated with higher risk of all-cause mortality and importantly, COPD was associated with fourfold higher risk of respiratory-related deaths in CKD population. The observed associations were more pronounced in younger CKD patients and among females with CKD.

Previous studies have examined the prevalence of $\mathrm{CKD}$ in COPD but population studies examining the prevalence of COPD in CKD population are lacking [10]. Our prevalence estimate is probably an underestimation, given the lack of spirometry data. It is important to note that even in general population, COPD is underdiagnosed with about $63 \%$ of adults showing evidence of impaired lung function but do not carry a diagnosis of lung disease such as asthma, chronic bron-
42

Am J Nephrol 2016;43:39-46

DOI: $10.1159 / 000444422$
Navaneethan/Schold/Huang/Nakhoul/ Jolly/Arrigain/Dweik/Nally Jr 
Table 2. Factors associated with COPD in those with CKD

\begin{tabular}{|c|c|c|c|}
\hline Effect & OR $(95 \% \mathrm{CI})^{*}$ & Effect & OR $(95 \% \mathrm{CI})^{*}$ \\
\hline Age (per 10 years increase) & $1.26(1.21-1.31)$ & Diabetes & $1.19(1.08-1.30)$ \\
\hline African American & $1.14(1.01-1.27)$ & Malignancy & $1.10(1.00-1.20)$ \\
\hline $\mathrm{BMI}, \mathrm{kg} / \mathrm{m}^{2}$ & & disease & $1.64(1.49-1.79)$ \\
\hline $18.5-24.9$ & Reference & Congestive heart failure & $2.30(2.06-2.57)$ \\
\hline $25-29.9$ & $0.80(0.72-0.89)$ & Hypertension & $3.02(2.51-3.63)$ \\
\hline CKD stage (eGFR), $\mathrm{ml} / \mathrm{min} / 1.73 \mathrm{~m}^{2}$ & & & \\
\hline $45-59$ & Reference & \multirow{3}{*}{\multicolumn{2}{|c|}{$\begin{array}{l}{ }^{*} \text { Adjusted for year of entry into the registry. ORs shown were } \\
\text { pooled using MIanalyze from } 5 \text { datasets created using multiple im- }\end{array}$}} \\
\hline $30-44$ & $0.97(0.89-1.07)$ & & \\
\hline $15-29$ & $0.86(0.73-1.01)$ & & \\
\hline
\end{tabular}

Fig. 1. Survival among those with and without COPD in CKD.

Fig. 2. Associations of various causes of death in those with COPD and CKD.
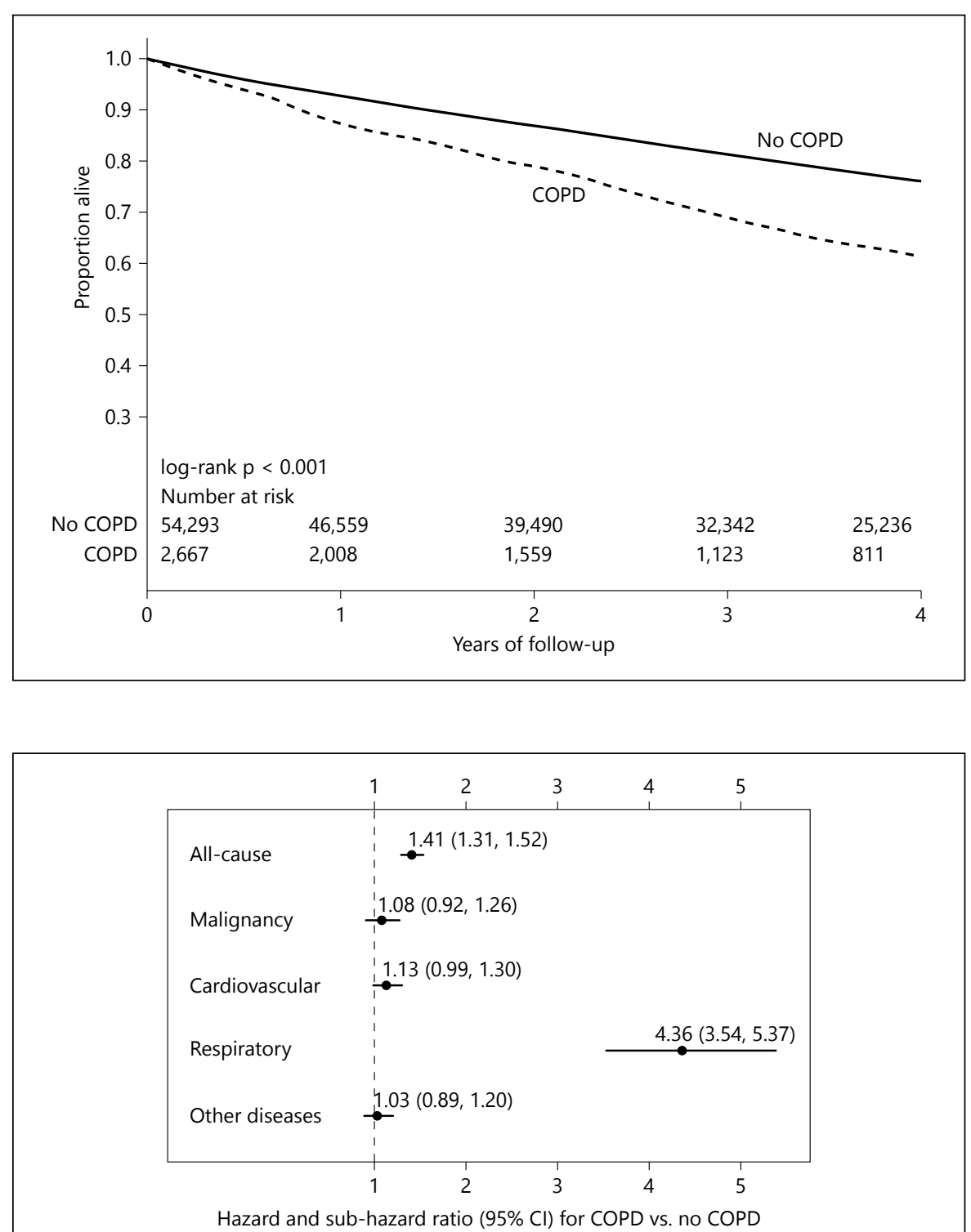

Am J Nephrol 2016;43:39-46 DOI: $10.1159 / 000444422$ 
those with COPD and CKD is unclear and merits further studies.

What might explain the higher hazards for overall and respiratory deaths in a CKD population? Higher infection-related deaths, particularly with pneumonia, have been reported in the CKD population and thus, the additional presence of COPD could further increase the risk for pneumonia or other infection due to immunosuppression from long-term use of COPD medications explaining the noted observations [18-21]. Also, COPD is a known contributor to pulmonary hypertension and could contribute to adverse outcomes in CKD [22]. Further, COPD by itself is associated with inflammation and is also accompanied by other chronic diseases (including CKD) that are also associated with systemic inflammation [23, 24]. Physical inactivity and metabolic syndrome are associated with both COPD and CKD [23, 25]. An inverse association between physical activity level and inflammation were noted in those with COPD. These factors could contribute to the excess mortality risk in those with COPD and CKD and warrant further investigation. We noted higher hazards for death in younger population with COPD. It appears that with aging, the effect of COPD on outcomes become less relevant in those with CKD. Even though females were not at higher odds of having COPD in CKD, its presence has more pronounced effects than in males. Mechanisms for such discrepant impact in unclear and require further studies.

In addition to mortality, morbidity and costs associated with care of patients with COPD and CKD are staggering. Data from a large claims database (Truven Health MarketScan Commercial Claims and Encounters and the MarketScan Medicare Supplemental Databases) showed that COPD- or asthma-related total health-care costs were greatest among patients with COPD and asthma and CKD ( $\$ 41,288$ for the first year after the diagnosis of CKD). Our results add to the existing literature about the mortality burden among this population and argue for further studies to elucidate the important clinical factors associated with COPD among those with CKD [26].

This study has several strengths. It is comprised of a large diverse clinical population of stages 3 and 4 CKD patients enhancing the generalizability of our results. Furthermore availability of both all-cause mortality and cause-specific death details in a population with CKD and COPD is novel. Additionally, we conducted several key sensitivity analyses and our results remained the same confirming the robustness of the findings. Nevertheless, as an observational study using a clinical population, there are important limitations. We included patients who are followed in our health system for various clinical conditions and hence whether these data can be applicable to less sicker community-based CKD population is unknown. We did not have spirometry data and previous studies have showed the limited utility of ICD-9 codes to define COPD and other lung diseases for such epidemiological investigations, but $[27,28]$ it is suggested that the use of multiple diagnostic codes for COPD and pharmacy data for medications can improve the ability to appropriately identify COPD patients [29]. However, even though we did not have pharmacy data, we did a sensitivity analyses by defining COPD as those who had the ICD- 9 codes and were on relevant medications and found similar results.

We acknowledge that it is a single-center study, limiting its potential for external validity, but such associations in a large CKD cohort have not been reported previously. We lacked comprehensive data for some variables, but performed multiple imputations and complete case analysis to address the 'missingness' issue, and overall, our findings remained the same. We obtained cause-specific death data from the State of Ohio Department of Health mortality files. The National Death Index obtains data from individual states, and several studies have reported using these data, which are considered reliable. For previous studies, we recently performed a validation study for those who died within our hospital system and this analysis confirmed the reliability of death certificate derived data [30]. The overlap syndrome of asthma and COPD has been consistently reported in studies with an estimated prevalence of $20 \%$ in patients with obstructive airways diseases [31]. We did not have spirometry data to distinguish this syndrome.

In summary, several demographic and clinical factors such as diabetes, heart failure, and hypertension were associated with COPD in stages 3 and 4 CKD population. The presence of COPD was associated with higher mortality in this population after accounting for other significant risk factors. Deaths due to respiratory disease accounted for a higher mortality burden among those with COPD and CKD. Additional studies to estimate the prevalence of COPD in CKD population using spirometry data to better characterize the lung disease and see if associations differ are needed. Fundamentally, additional research is needed to elucidate the mechanisms that link COPD and CKD that could then lead to important targeted therapy at improving the outcomes of those with COPD and CKD. 


\section{Grant Support and Disclosure}

S.D.N. is supported by a grant from the National Institutes of Health (NIH, R01DK101500); J.V.N. is supported by a grant from the NIH (DK094112) and S.E.J. is supported by a grant from the $\mathrm{NIH}$ (K23DK091363). The content is solely the responsibility of the authors and does not necessarily represent the official views of the NIH. The creation of the CCF CKD registry was funded by an unrestricted grant from Amgen, Inc., to the Department of Nephrology and Hypertension Research and Education Fund. The results presented in this paper have not been published previously in whole or part.

\section{Acknowledgments}

The authors wish to thank Welf Saupe, Vicky Konig, and John Sharp of Cleveland Clinic who helped in data extraction during the development of the registry.

\section{Disclosure Statement}

The authors have no financial interests relevant to the contents of this manuscript.

\section{References}

1 Mannino DM, Buist AS: Global burden of COPD: risk factors, prevalence, and future trends. Lancet 2007;370:765-773.

2 Agarwal S, Rokadia H, Senn T, Menon V: Burden of cardiovascular disease in chronic obstructive pulmonary disease. Am J Prev Med 2014;47:105-114.

3 Go AS, Chertow GM, Fan D, McCulloch CE, Hsu CY: Chronic kidney disease and the risks of death, cardiovascular events, and hospitalization. N Engl J Med 2004;351:1296-1305.

4 Gupta J, Mitra N, Kanetsky PA, Devaney J, Wing MR, Reilly M, et al: Association between albuminuria, kidney function, and inflammatory biomarker profile in CKD in CRIC. Clin J Am Soc Nephrol 2012;7:19381946.

5 van Gestel YR, Chonchol M, Hoeks SE, Welten GM, Stam H, Mertens FW, et al: Association between chronic obstructive pulmonary disease and chronic kidney disease in vascular surgery patients. Nephrol Dial Transplant 2009;24:2763-2767.

6 Incalzi RA, Corsonello A, Pedone C, Battaglia S, Paglino G, Bellia V, et al: Chronic renal failure: a neglected comorbidity of COPD. Chest 2010;137:831-837.

7 Chandra D, Stamm JA, Palevsky PM, Leader JK, Fuhrman CR, Zhang Y, et al: The relationship between pulmonary emphysema and kidney function in smokers. Chest 2012;142: 655-662.

8 Funakoshi Y, Omori H, Mihara S, Onoue A, Ogata Y, Aizawa H, et al: C-reactive protein levels, airflow obstruction, and chronic kidney disease. Environ Health Prev Med 2012; 17:18-26.

9 Navaneethan SD, Jolly SE, Schold JD, Arrigain S, Saupe W, Sharp J, et al: Development and validation of an electronic health recordbased chronic kidney disease registry. Clin J Am Soc Nephrol 2011;6:40-49.

10 Ford ES: Urinary albumin-creatinine ratio, estimated glomerular filtration rate, and allcause mortality among US adults with obstructive lung function. Chest 2015;147:56-67.

11 Lamprecht B, Soriano JB, Studnicka M, Kaiser B, Vanfleteren LE, Gnatiuc L, et al: Determi- nants of underdiagnosis of COPD in national and international surveys. Chest 2015;148: 971-985.

12 Abramson MJ, Schattner RL, Sulaiman ND, Del Colle EA, Aroni R, Thien F: Accuracy of asthma and COPD diagnosis in Australian general practice: a mixed methods study. Prim Care Respir J 2012;21:167-173.

13 Afonso AS, Verhamme KM, Sturkenboom MC, Brusselle GG: COPD in the general population: prevalence, incidence and survival. Respir Med 2011;105:1872-1884.

14 Leivseth L, Brumpton BM, Nilsen TI, Mai XM, Johnsen R, Langhammer A: GOLD classifications and mortality in chronic obstructive pulmonary disease: the HUNT study, Norway. Thorax 2013;68:914-921.

15 Miniati M, Monti S, Pavlickova I, Bottai M: Survival in COPD: impact of lung dysfunction and comorbidities. Medicine (Baltimore) 2014;93:e76.

16 Pływaczewski R, Maciejewski J, Bednarek M, Zieliński J, Górecka D, Śliwiński P: Causes of deaths in COPD patients in primary care setting - a 6-year follow-up. Pneumonol Alergol Pol 2015;83:193-202.

17 Lahousse L, Niemeijer MN, van den Berg ME, Rijnbeek PR, Joos GF, Hofman A, et al: Chronic obstructive pulmonary disease and sudden cardiac death: the Rotterdam study. Eur Heart J 2015;36:1754-1761.

18 Chou CY, Wang SM, Liang CC, Chang CT, Liu JH, Wang IK, et al: Risk of pneumonia among patients with chronic kidney disease in outpatient and inpatient settings: a nationwide population-based study. Medicine (Baltimore) 2014;93:e174.

19 Dalrymple LS, Go AS: Epidemiology of acute infections among patients with chronic kidney disease. Clin J Am Soc Nephrol 2008;3: 1487-1493.

20 Sethi S, Murphy TF: Infection in the pathogenesis and course of chronic obstructive pulmonary disease. N Engl J Med 2008;359: 2355-2365.

21 McDonald HI, Thomas SL, Millett ER, Nitsch $\mathrm{D}$ : CKD and the risk of acute, communityacquired infections among older people with diabetes mellitus: a retrospective cohort study using electronic health records. Am J Kidney Dis 2015;66:60-68.

22 Navaneethan SD, Roy J, Tao K, Brecklin CS, Chen J, Deo R, et al: Prevalence, predictors, and outcomes of pulmonary hypertension in CKD. J Am Soc Nephrol 2015; pii: ASN.2014111111.

23 Loprinzi PD, Walker JF, Lee H: Association between physical activity and inflammatory markers among U.S. adults with chronic obstructive pulmonary disease. Am J Health Promot 2014;29:81-88.

24 Magnussen H, Watz H: Systemic inflammation in chronic obstructive pulmonary disease and asthma: relation with comorbidities. Proc Am Thorac Soc 2009;6:648-651.

25 Navaneethan SD, Schold JD, Kirwan JP, Arrigain S, Jolly SE, Poggio ED, et al: Metabolic syndrome, ESRD, and death in CKD. Clin J Am Soc Nephrol 2013;8:945-952.

26 Mannino DM, Higuchi K, Yu TC, Zhou H, Li $\mathrm{Y}$, Tian $\mathrm{H}$, et al: Economic burden of COPD in the presence of comorbidities. Chest 2015; 148:138-150.

27 Quint JK, Müllerova H, DiSantostefano RL, Forbes H, Eaton S, Hurst JR, et al: Validation of chronic obstructive pulmonary disease recording in the clinical practice research datalink (CPRD-GOLD). BMJ Open 2014;4: e005540.

28 Molnar MZ, Mucsi I, Novak M, Szabo Z, Freire AX, Huch KM, et al: Association of incident obstructive sleep apnoea with outcomes in a large cohort of US veterans. Thorax 2015;70:888-895.

29 Cooke CR, Joo MJ, Anderson SM, Lee TA, Udris EM, Johnson E, et al: The validity of using ICD-9 codes and pharmacy records to identify patients with chronic obstructive pulmonary disease. BMC Health Serv Res 2011;11:37.

30 Navaneethan SD, Schold JD, Arrigain S, Jolly SE, Nally JV Jr: Cause-specific deaths in nondialysis-dependent CKD. J Am Soc Nephrol 2015;26:2512-2520.

31 Gibson PG, McDonald VM: Asthma-COPD overlap 2015: now we are six. Thorax 2015;70: 683-691. 